\title{
ASSOCIAÇÕES ENTRE O TEMPO DE EXPERIÊNCIA PROFISSIONAL E A NOMENCLATURA DOS EXERCÍCIOS UTILIZADAS NO TREINAMENTO DE FORÇA
}

\author{
ASSOCIATIONS BETWEEN PROFESSIONAL TIME EXPERIENCE \\ AND NOMENCLATURE ADOPTED IN STRENGTH TRAINING
}

\section{Leonardo Mendes Leal de Souza ${ }^{\mathrm{a}^{*}}$, Gabriel Andrade Paz ${ }^{\mathrm{b}^{*}}$, Viviane Schultz Straatmann ${ }^{\mathrm{c}^{* *}}$, Humberto Miranda ${ }^{\mathrm{d}^{*}}$}

\author{
aleomendes84@gmail.com, bgabriel.andrade.paz@gmail.com, cvica_s@hotmail.com, dhumbertomiranda01@gmail.com \\ *Universidade Federal do Rio de Janeiro - Rio de Janeiro (RJ), Brasil \\ **Universidade do Estado do Rio de Janeiro - Rio de Janeiro (RJ), Brasil
}

Data de recebimento do artigo: 20/05/2016 Data de aceite do artigo: 28/09/2016

\section{RESUMO}

Introdução: A divergência em relação à nomenclatura dos exercícios adotada por professores de educação física no âmbito profissional pode gerar sérios problemas de comunicação e consequentemente interferir na prescrição e qualidade do treinamento proposto. Objetivo: Verificar a associação entre o tempo de experiência no âmbito profissional com a nomenclatura de exercícios do treinamento de força adotados por profissionais de educação física. Materiais e métodos: Participaram da pesquisa 191 voluntários. O teste qui-quadrado foi aplicado para verificar a associação entre as variáveis. Resultados: Foram observadas diferenças significativas entre as opçōes de nomenclaturas para nove dos dez exercícios apresentados. No entanto, nenhum dos exercícios analisados apresentou associação entre as opçóes de nomenclatura de resposta com o tempo de experiência em treinamento de força. Conclusáo: $\mathrm{O}$ presente estudo evidencia a padronização dos nomes de nove dos dez exercícios por profissionais de educação física independentemente do tempo de sua experiência profissional.

Palavras-chave: Exercício; treinamento de força; cinesiologia; nomenclatura.

\section{ABSTRACT}

Introduction: The divergences of the nomenclature of exercises adopted by physical education teachers in their professional environment can lead to serious communication problems, and consequently interfere in the prescription and the quality of the proposed training. Objective: To associate time of professional experience with the strength training nomenclatures selected by physical education professionals, and identify whether there are differences between the naming frequencies selected for the exercises adopted. Materials and methods: The current study included 191 volunteers. The Chi square test was applied to verify the association of the variables. Results: Significant differences were observed between the nomenclatures options for nine out of ten exercises presented. Nevertheless, there was no association between the responses of nomenclature options with professional experience time in strength training. Conclusion: The present study shows a pattern of names in nine out of ten exercises from physical education professionals independently of their professional experience time.

Keywords: Exercise; strength training; kinesiology; nomenclature. 


\section{Introdução}

A nomenclatura ou terminologia acerca dos exercícios é determinada a partir de movimentos articulares em seus respectivos planos anatômicos de referência ${ }^{1}$. No entanto, a padronização de nomenclatura dos exercícios realizados no treinamento de força (TF) ainda é uma temática pouco abordada na literatura científica, sendo assim um fator limitante para comunicaçáo entre estudantes, profissionais e praticantes. Apesar desse panorama, nos últimos anos algumas pesquisas têm demonstrado maior interesse em investigar esse tema, permitindo, desse modo, o início de uma maior reflexão sobre esse assunto ${ }^{2-5}$.

O TF, também conhecido como treinamento resistido, tem sido uma das formas mais populares de exercício físico aplicado na melhora do desempenho de atletas e na aptidão física e funcional da população em geral ${ }^{6}$. Esse tipo de treinamento refere-se a um método especializado de condicionamento físico e envolve a combinação de diferentes variáveis metodológicas, como carga, velocidade de execução e tempo de intervalo, assim como a execuçáo de exercícios utilizando o peso corporal, máquinas, elásticos ou pesos livres ${ }^{7}$.

Faulkner ${ }^{8}$ apresentou em seu estudo as dificuldades relacionadas ao uso inadequado de terminologias relacionadas às contraçóes musculares em uma ação dinâmica ou estática, destacando também a necessidade de uma comunicação clara e precisa para um entendimento imediato entre os pesquisadores da área. Tal condição pode se tornar um fator limitante na prática profissional, considerando que usualmente fica a critério do profissional determinar a nomenclatura e padrão de execução do exercício.

$\mathrm{Na}$ própria literatura científica frequentemente são observados diferentes nomes para um mesmo exercício. Com esse objetivo, Jackson et al. ${ }^{2}$, através da aplicação de um questionário, verificaram a inconsistência nas nomenclaturas adotadas para exercícios comumente usados no TF. No entanto, esse estudo levou em consideração profissionais especializados em diferentes áreas da saúde, como universitários, médicos, personal trainers, treinadores e preparadores físicos. A partir dos resultados obtidos nesse estudo, os autores ressaltam a necessidade de estabelecer um padrão de nomenclatura, haja vista a variedade de nomes coletados para cada tipo de exercício por esses indivíduos. Adicionalmente, a mesma inconsistência sobre a padronização pode ser observada entre profissionais das áreas de educação física e fisioterapia, ao responderem sobre as nomenclaturas utilizadas do método pilates ${ }^{3}$. Por outro lado, buscando também investigar essa padronização dos nomes utilizados no TF, em estudo de Souza et al. ${ }^{4}$, os autores identificaram um padrão de respostas para os dez exercícios pesquisados, contribuindo dessa forma para um avanço no sentido da denominaçáo padronizada de determinados exercícios.

Contudo, até o presente momento náo foram identificadas evidências na literatura que relacionem o tempo de experiência no âmbito profissional em associação à nomenclatura adotada para os exercícios de força. Além disso, a divergência em relação à nomenclatura adotada na prática profissional pode gerar sérios problemas de comunicação e consequentemente interferir na prescrição e qualidade do treinamento proposto. Portanto, este estudo tem como objetivo verificar a relação entre o tempo de experiência profissional com TF e as nomenclaturas de exercícios adotadas por esses profissionais, tendo como hipótese que o tempo de experiência interfere na escolha da nomenclatura.

\section{Materiais e métodos}

\section{Participantes}

O estudo incluiu 191 participantes, sendo 130 homens e 61 mulheres, estudantes de graduação e profissionais de educação física com experiência em TF, de academias das regióes norte, sul, oeste e centro do município do Rio de Janeiro (RJ), entre agosto de 2013 e maio de 2014. O delineamento transversal foi adotado com amostra selecionada por conveniência, não probabilística, assumindo os seguintes critérios de inclusão: a) ser um estudante de graduação ou profissional de educação física; b) quando profissionais, terem trabalhado com TF por no mínimo um ano, antecedendo o início do estudo; c) quando estudantes de graduação, terem passado por programa de estágio e/ou treinamento supervisionado com TF por no mínimo um ano. Os indivíduos que não atingiram os critérios citados acima foram excluídos do estudo.

Foi solicitado a todos os participantes que lessem e assinassem o termo de consentimento livre e esclarecido antes de iniciarem qualquer procedimento do estudo. Este estudo foi aprovado pelo Comitê de Ética em Pesquisa da instituição Universidade Federal do Rio de Janeiro sob o processo no 43249815.4.0000.5257, de acordo com a Resolução 466/12 do Conselho Nacional de Saúde e da Declaração de Helsinki.

\section{Procedimentos}

Considerando a ausência de um instrumento validado para avaliar a padronização das nomenclaturas de exercícios de força, o instrumento adotado por Jackson 
et al. $^{2}$ foi adaptado adotando dez exercícios de força ilustrados, sendo dois de membros inferiores e oito de membros superiores, utilizados com frequência no ambiente de TF para compor o questionário utilizado. Esses exercícios foram selecionados pelo fato de ainda não terem sido abordados em estudos com essa temática e ainda causarem divergências em suas nomenclaturas.

Dessa forma, duas imagens, uma com a posição inicial e outra com a posição final dos exercícios, foram apresentadas aos participantes (Figuras 1 e 2). $\mathrm{O}$ respondente tinha como alternativa de resposta opçóes fechadas (com as nomenclaturas mais usuais, incluindo uma resposta-referência baseada na literatura, não apresentada ao respondente) e uma opção aberta, caso as alternativas de resposta fechada não atendessem a sua necessidade de resposta. Juntamente a esse questionário, perguntas sobre informaçóes gerais, como idade, região de trabalho, formação acadêmica e tempo de experiência com treinamento de força (TETF) foram preenchidas pelos indivíduos. Inicialmente, foi exposta aos participantes a importância do estudo para comunicação entre os profissionais e como essa questão poderia influenciar diretamente ou indiretamente em sua atividade laboral diária.

Figura 1: Ilustração dos exercícios 1 ao 6 com as suas respectivas posições iniciais (A) e finais (B) do movimento.

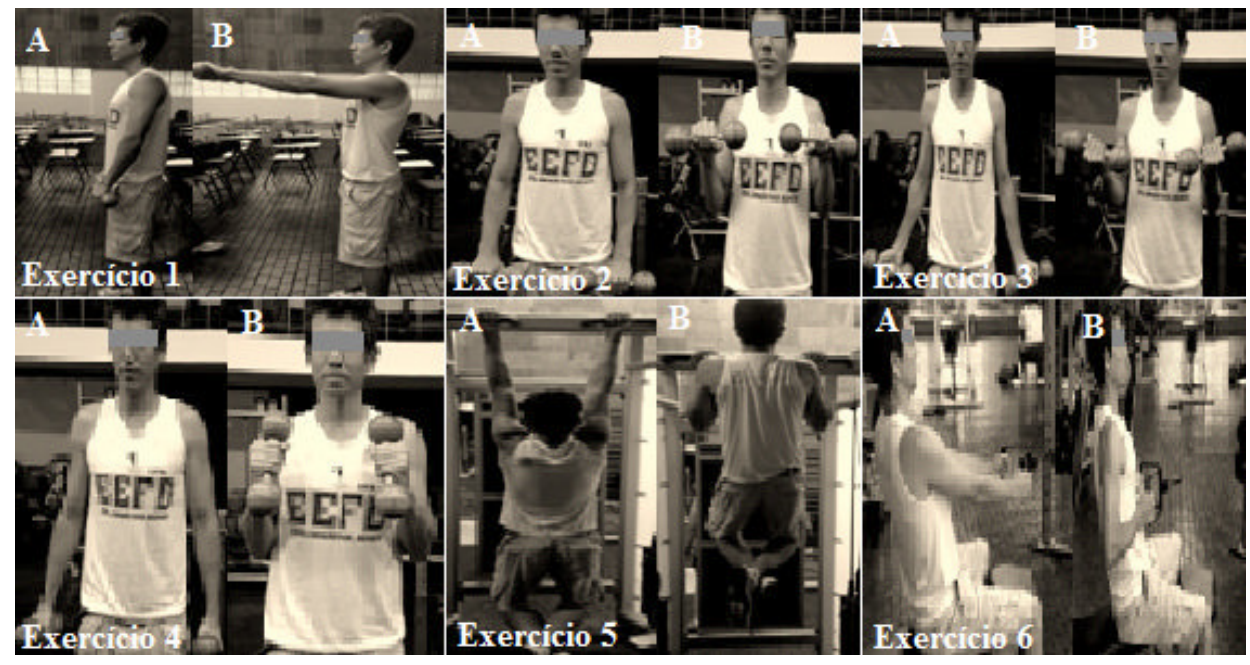

Figura 2: Ilustração dos exercícios 7 ao 10 com as suas respectivas posições inicial (A) e final (B) do movimento.

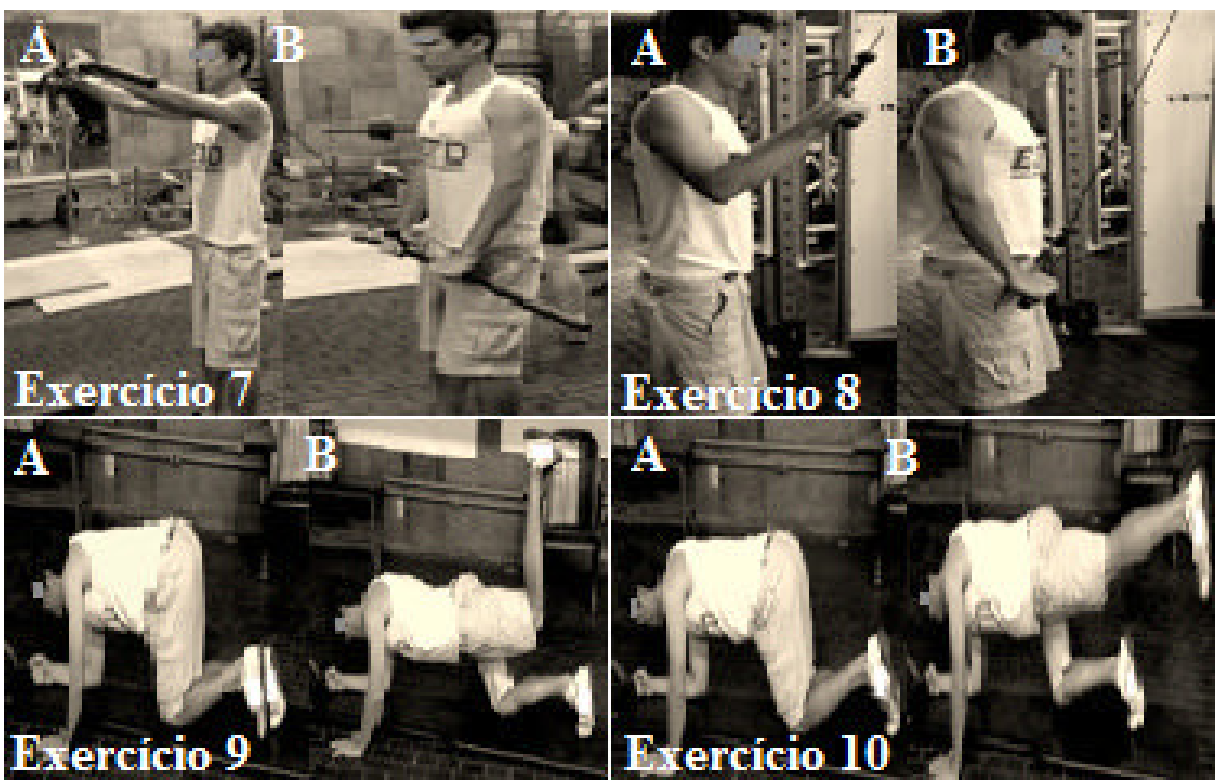




\section{Tratamento estatístico}

Utilizou-se abordagem quantitativa do tipo descritiva e inferencial para o tratamento estatístico do presente manuscrito. Inicialmente foi verificada a normalidade das variáveis pelo teste Kolmogorov-Smirnov. Dada a distribuição normal das variáveis de interesse, os procedimentos estatísticos adotados para caracterizar a amostra foram médios, com desvio-padrão para variáveis contínuas e frequências para variáveis categóricas por gênero. $\mathrm{O}$ teste $\mathrm{T}$ para amostras independentes (variáveis contínuas) e qui-quadrado (variáveis categóricas) foram aplicados para verificar associações entre as variáveis de interesse entre sexos. Ainda para fins de caracterização da amostra, foi realizado o teste de Pearson para verificar a correlação entre os escores $Z$ (padronização de medidas) das variáveis idade (em anos) e TETF (em meses). Para analisar a associação entre a nomenclatura adotada para cada um dos exercícios de força e TETF (em meses), foi utilizado o teste qui-quadrado ou o teste de Fisher, quando adequado. O teste qui-quadrado particionado foi utilizado para verificar diferenças entre as proporçóes de resposta de cada exercício.

A variável TETF (em meses) foi dividida em quartis; as opçóes de resposta para os dez exercícios analisados foram trabalhadas em categorias de resposta com os nomes mais usuais em ambientes de TF e na literatura. As opçóes de respostas abertas, chamadas de "outras", foram uma alternativa para o respondente colocar sua nomenclatura utilizada se não estivesse entre as demais opçóes fornecidas no questionário. Os procedimentos estatísticos foram realizados no software SPSS 20.0, e o nível de significância adotado foi $\mathrm{p} \leq 0,05$.

\section{Resultados}

Dentre os participantes do estudo, $67,8 \%$ são do sexo masculino, com idade média de 27 anos (homens: $27,8 \pm 6,5$; mulheres: $27 \pm 7,6)$ para ambos os sexos. A média de TETF entre os homens foi maior do que entre as mulheres $(56,4 \pm 56,9 ; 43,4 \pm 60,5)$, sem diferença estatisticamente significativa. A proporção de profissionais e de estudantes por sexo não variou, apresentando frequências próximas a 50\% (Tabela 1).

Analisou-se o TETF (meses) por quartis, sendo que para a amostra como um todo, os percentis 25,50 e 75 corresponderam aos valores 12,36 e 60 , respectivamente, com valores no mínimo de 3 e no máximo de 384 . A correlação de Pearson foi realizada entre o escore $Z$ das variáveis TETF (meses) e idade (anos), podendo-se verificar uma correlação positiva e significativa $(r=0,756$; $\mathrm{p}<0,001)$ (dados não apresentados em tabela). Foi observada diferença estatisticamente significativa entre os quartis de TETF por gêneros ( $\mathrm{p}=0,008)$; entre as mulheres a maior proporção se concentrou no $2^{\circ}$ quartil; para os homens, no $4^{\circ}$ quartil. Entre as regióes, para ambos os sexos, a região norte foi a que apresentou maior proporção de indivíduos, sem diferenças estatisticamente significativas (Tabela 1).

Tabela 1: Caracterização da amostra com variáveis sociodemográficas, grau acadêmico e tempo de experiência com treinamento de força, por sexo. Rio de Janeiro-RJ, 2013-2014.

\begin{tabular}{|c|c|c|c|}
\hline Variáveis & \multicolumn{2}{|c|}{ Sexo1 } & \\
\hline & $\begin{array}{c}\text { Masculino } \\
(\mathrm{N}=122)\end{array}$ & $\begin{array}{c}\text { Feminino } \\
(\mathrm{N}=59)\end{array}$ & \\
\hline & \multicolumn{2}{|c|}{ Média ( $\pm \mathrm{DP})$} & Valor de $\mathrm{p}^{2}$ \\
\hline Idade (anos) & $27,8( \pm 6,5)$ & $27,0( \pm 7,6)$ & 0,408 \\
\hline \multirow{2}{*}{ TETF (meses) } & $56,4( \pm 56,9)$ & $43,4( \pm 60,5)$ & 0,164 \\
\hline & & & Valor de $\mathrm{p}^{3}$ \\
\hline Grau acadêmico & & & 0,839 \\
\hline Profissionais & $52,2(64)$ & $50,8(30)$ & \\
\hline Estudantes & $47,5(58)$ & $49,2(29)$ & \\
\hline Quartil de TETF & & & $0,008^{*}$ \\
\hline $1^{\circ}$ quartil & $24,6(30)$ & $36,2(21)$ & \\
\hline $2^{\circ}$ quartil & $24,6(30)$ & $39,7(23)$ & \\
\hline $3^{\circ}$ quartil & $20,5(25)$ & $12,1(7)$ & \\
\hline $4^{\circ}$ quartil & $30,3(37)$ & $12,1(7)$ & \\
\hline Regiáo do Rio de Janeiro & & & 0,843 \\
\hline Norte & $29,9(35)$ & $34,5(19)$ & \\
\hline Sul & $23,9(28)$ & $18,2(10)$ & \\
\hline Oeste & $17,9(21)$ & $18,2(10)$ & \\
\hline Centro & $28,2(33)$ & $29,1(16)$ & \\
\hline
\end{tabular}

${ }^{1} 10$ dados faltantes para sexo; ${ }^{2}$ Teste $\mathrm{T}$ para amostras independentes; ${ }^{3}$ Teste qui-quadrado; ${ }^{*}$ diferença estatisticamente significativa $(\mathrm{p}<0,05)$. 
Na Tabela 2 foram apresentados os resultados referentes à investigação da associação entre as nomenclaturas dos exercícios de força e o TETF. Nenhum dos exercícios analisados apresentou associação entre as opçóes de nomenclatura de resposta com o TETF. Todos os exercícios com exceção do número 6 apresentaram diferenças estatisticamente significativas entre os percentuais de resposta de cada categoria, independentemente do tempo de experiência profissional.

Tabela 2: Frequência de resposta nas opções de nomenclatura de cada exercício por quartil de tempo de experiência com treinamento de força (TETF). Rio de Janeiro-RJ, 2013-2014.

\begin{tabular}{|c|c|c|c|c|c|c|c|}
\hline \multirow[t]{2}{*}{ Exercícios } & \multirow[t]{2}{*}{ Nomenclatura } & \multicolumn{4}{|c|}{ TETF } & \multirow[t]{2}{*}{ Valor de $p$} & \multirow[t]{2}{*}{$\begin{array}{c}\text { Total } \\
(\%)\end{array}$} \\
\hline & & $\begin{array}{l}1^{\circ} Q \text { uartil } \\
\text { TETF (\%) }\end{array}$ & $\begin{array}{l}2^{\circ} \text { Quartil } \\
\text { TETF (\%) }\end{array}$ & $\begin{array}{l}3^{\circ} \text { Quartil } \\
\text { TETF (\%) }\end{array}$ & $\begin{array}{l}4^{\circ} \text { Quartil } \\
\text { TETF (\%) }\end{array}$ & & \\
\hline \multirow{4}{*}{$\begin{array}{c}1 \\
N=191\end{array}$} & Flexão de ombro ${ }^{2}$ & $54,5 \%$ & $53,7 \%$ & $54,3 \%$ & $34,8 \%$ & \multirow{4}{*}{$0,167^{3}$} & 49,4 \\
\hline & Elevação frontal & $27,3 \%$ & $20,4 \%$ & $14,3 \%$ & $23,9 \%$ & & 22,1 \\
\hline & Flexão de ombros pronada & $16,4 \%$ & $25,9 \%$ & $31,4 \%$ & $39,1 \%$ & & 27,4 \\
\hline & Outro & $1,8 \%$ & $0 \%$ & $0 \%$ & $2,2 \%$ & & 1,1 \\
\hline \multirow{4}{*}{$\begin{array}{c}2 \\
N=190\end{array}$} & Bíceps invertido $^{2}$ & $38,2 \%$ & $55,6 \%$ & 50 & 52,2 & \multirow{4}{*}{$0,096^{3}$} & 48,7 \\
\hline & Bíceps direto pegada pronada & $50,8 \%$ & $31,5 \%$ & 29,4 & 23,9 & & 34,9 \\
\hline & Flexão de cotovelos pronada & $5,5 \%$ & $11,1 \%$ & 11,8 & 19,6 & & 11,6 \\
\hline & Outro & $5,5 \%$ & $1,9 \%$ & 8,8 & 4,3 & & 4,8 \\
\hline \multirow{4}{*}{$\begin{array}{c}3 \\
N=191\end{array}$} & Rosca bíceps direta ${ }^{2}$ & 63,6 & 61,1 & 60 & 60,9 & \multirow{4}{*}{$0,742^{3}$} & 61,6 \\
\hline & Bíceps direto pegada supinada & 29,1 & 24,1 & 20 & 23,9 & & 24,7 \\
\hline & Flexáo de cotovelos supinada & 3,6 & 11,1 & 11,4 & 13 & & 9,5 \\
\hline & Outro & 3,6 & 3,7 & 8,6 & 2,2 & & 4,2 \\
\hline \multirow{4}{*}{$\begin{array}{c}4 \\
N=189\end{array}$} & Bíceps direto pegada neutra ${ }^{2}$ & 58,2 & 62,3 & 68,8 & 51,1 & \multirow{4}{*}{$0,093^{3}$} & 59,6 \\
\hline & Bíceps martelo & 32,7 & 32,1 & 17,1 & 26,7 & & 28,1 \\
\hline & Flexão de cotovelos neutra & 3,6 & 5,7 & 14,3 & 17,8 & & 9,6 \\
\hline & Outro & 5,5 & 0 & 0 & 4,4 & & 2,7 \\
\hline \multirow{4}{*}{$\begin{array}{c}5 \\
N=191\end{array}$} & Barra fixa supinada ${ }^{2}$ & 47,3 & 48,1 & 40 & 39,1 & \multirow{4}{*}{$0,150^{3}$} & 44,2 \\
\hline & Puxada supinada na barra & 25,5 & 38,9 & 42,9 & 47,8 & & 37,9 \\
\hline & Puxada na barra fixa & 27,3 & 13 & 14,3 & 10,9 & & 16,8 \\
\hline & Outro & 0 & 0 & 2,9 & 2,2 & & 1,1 \\
\hline \multirow{4}{*}{$\begin{array}{c}6 \\
N=189\end{array}$} & Remada dorsal fechada $^{2}$ & 33,3 & 24,1 & 32,4 & 26,1 & \multirow{4}{*}{$0,275^{1}$} & 28,7 \\
\hline & Remada baixa & 38,9 & 25,9 & 20,6 & 28,3 & & 29,3 \\
\hline & Remada aduzida neutra & 16,7 & 14,8 & 14,7 & 15,2 & & 15,4 \\
\hline & Outro & 11,1 & 35,2 & 32,4 & 30,4 & & 26,6 \\
\hline \multirow{4}{*}{$\begin{array}{c}7 \\
\mathbf{N}=\mathbf{1 8 7}\end{array}$} & Extensão de ombros ${ }^{2}$ & 23,6 & 32,1 & 21,9 & 26,1 & \multirow{4}{*}{$0,122^{3}$} & 26,3 \\
\hline & Crossover na polia alta & 20 & 5,7 & 0 & 6,5 & & 9,1 \\
\hline & Extensão de ombros na polia alta & 47,3 & 54,7 & 62,5 & 60,9 & & 55,4 \\
\hline & Outro & 9,1 & 7,5 & 15,6 & 6,5 & & 9,1 \\
\hline \multirow{4}{*}{$\begin{array}{c}8 \\
N=190\end{array}$} & Tríceps no pulley ${ }^{2}$ & 34,5 & 38,9 & 29,4 & 43,5 & \multirow{4}{*}{$0,586^{3}$} & 37 \\
\hline & Tríceps no cross & 49,1 & 38,9 & 44,1 & 30,4 & & 40,7 \\
\hline & $\begin{array}{l}\text { Extensão de cotovelos na polia } \\
\text { alta }\end{array}$ & 10,9 & 18,5 & 14,7 & 15,2 & & 14,8 \\
\hline & Outro & 5,5 & 3,7 & 11,8 & 10,9 & & 7,4 \\
\hline \multirow{4}{*}{$\begin{array}{c}9 \\
N=189\end{array}$} & $\begin{array}{c}\text { Glúteos } 3 \text { apoios com joelho } \\
90^{\mathrm{o}^{2}}\end{array}$ & 41,8 & 20,8 & 29,4 & 30,4 & \multirow{4}{*}{$0,228^{3}$} & 30,9 \\
\hline & $\begin{array}{l}\text { Extensão de quadril } 3 \text { apoios } \\
\text { com joelho } 90^{\circ}\end{array}$ & 47,3 & 66,0 & 61,8 & 58,7 & & 58,0 \\
\hline & $\begin{array}{c}\text { Glúteos } 3 \text { apoios com joelho } \\
\text { flexionado }\end{array}$ & 5,5 & 13,2 & 8,8 & 8,7 & & 9 \\
\hline & Outro & 5,5 & 0 & 0 & 2,2 & & 2,1 \\
\hline
\end{tabular}


Tabela 2: Continuação

\begin{tabular}{|c|c|c|c|c|c|c|c|}
\hline Exercícios & Nomenclatura & \multicolumn{4}{|c|}{ TETF } & Valor de $\mathrm{p}$ & $\begin{array}{c}\text { Total } \\
(\%)\end{array}$ \\
\hline \multirow{4}{*}{$\begin{array}{c}10 \\
N=190\end{array}$} & $\begin{array}{c}\text { Glúteos } 3 \text { apoios com extensão } \\
\text { de joelho }{ }^{2}\end{array}$ & 20,0 & 18,5 & 26,5 & 28,3 & \multirow{4}{*}{$0,251^{3}$} & 22,8 \\
\hline & $\begin{array}{l}\text { Extensão de quadril } 3 \text { apoios } \\
\text { com extensão de joelho }\end{array}$ & 54,5 & 64,8 & 64,7 & 58,7 & & 60,3 \\
\hline & Glúteos 3 apoios coice & 20 & 16,7 & 8,8 & 6,5 & & 13,8 \\
\hline & Outro & 5,5 & 0 & 0 & 6,5 & & 3,2 \\
\hline
\end{tabular}

${ }^{1}$ Teste qui-quadrado; ${ }^{2}$ Resposta referência; ${ }^{3}$ Teste de Fisher.

\section{Discussão}

A partir da hipótese de que o possível tempo de experiência pudesse interferir na escolha da nomenclatura, o presente estudo investigou a associação entre as nomenclaturas dos exercícios de força e o TETF. Diferentemente do esperado, não foi observado, para os exercícios selecionados, relação entre o TETF e os nomes adotados pelos profissionais de educação física, sugerindo que, independente da experiência profissional, os nomes adotados para os exercícios demonstraram uma homogeneidade nas escolhas entre os grupos analisados.

Dos resultados gerais, no caso da verificação da padronização da nomenclatura, todos os exercícios com exceção do número 6 apresentaram diferenças estatisticamente significativas entre os percentuais de resposta de cada categoria, independente do tempo de experiência profissional. Em contrapartida ao resultado encontrado no presente estudo, evidências prévias sugerem uma divergência quanto à nomenclatura de exercícios adotados no âmbito do TF. Nuzzo ${ }^{5}$, ao analisar o padrão de nomenclatura para os exercícios em geral, investigou cinquenta e sete exercícios descritos no manual da National Strength \& Conditioning Association ${ }^{9}$, sobre as técnicas dos exercícios do TF, e verificou que existiam trinta e cinco diferentes padrōes de nomenclaturas. $\mathrm{O}$ autor também descreve que nenhum padrão de nomenclatura foi identificado em mais de 4 exercícios, revelando dessa forma que as estratégias para as nomeaçóes dos exercícios ainda são inconsistentes.

Em estudo realizado por Jackson et al. ${ }^{2}$, foi aplicado um questionário com 10 exercícios típicos de TF para diversos profissionais da área de saúde, como personal trainers, treinadores, preparadores físicos, universitários e médicos. Houve uma variedade de opçóes tanto no que diz respeito à nomenclatura escolhida para o exercício quanto à escolha diferenciada entre esses profissionais. Os autores sugeriram uma padronização dos exercícios sem comprometer a criatividade dos exercícios prescritos pelos profissionais, beneficiando todos os envolvidos no treinamento e facilitando a comunicação.

$\mathrm{O}$ resultado encontrado para as diferentes nomenclaturas citadas no questionário pode ser uma consequência das diferentes linguagens: a de pesquisa ou científica, que é caracterizada por uma linguagem formal e codificada, e a linguagem da prática profissional, que é descrita como cotidiana e informal ${ }^{10}$. No entanto, nota-se que mesmo na literatura científica diferentes autores adotam variados nomes para o mesmo exercício, como "rosca tríceps no pulley" 11 " "rosca tríceps no puxador alto" ${ }^{12}$ ou simplesmente "tríceps pulley"13. Tal situação foi exemplificada no presente estudo no exercício 8 do questionário, no qual a opção que obteve maior número de respostas foi a que apresentou outra variação, "tríceps no cross". Outro exemplo é a utilização da abdução de ombro ${ }^{14}$ e elevação lateral ${ }^{15}$. Exercícios multiarticulares também podem ser encontrados contendo essas variaçóes, como no caso da remada unilateral com halteres ${ }^{16}$, remada fechada curvada unilateral ${ }^{17}$, ou somente remada curvada ${ }^{18}$. Essas distinçóes evidenciam o desafio de definir uma possível padronização para os exercícios em questão.

Além da escolha dos nomes dos exercícios serem evidentes em $90 \%$ dos movimentos analisados no presente estudo, pode-se perceber também certo consenso na literatura no campo da cinesiologia ao descrever um movimento. Por exemplo: um movimento articular de ombro, no plano sagital, elevando o braço anteriormente, é denominado "flexão de ombro"1,19,20, opção de resposta que pode ser observada no exercício 1 do presente estudo. Barbanti et al. ${ }^{21}$ propóem que haja uma maior integração entre as evidências teóricas e a prática do treinamento, desenvolvendo assim o entendimento científico com linguagens claras e naturais, na tentativa de diminuir a distância entre a investigação científica e a prática do treinamento.

Informaçôes sobre aparelho ou equipamento utilizado e tipo de empunhadura (supinada, pronada ou neutra) também são necessárias para uma boa comunicação tanto entre os professores como para os alunos. Na literatura científica é possível observar diversos nomes de exercícios que carecem de informaçóes complementares, por exemplo no exercício comumente chamado de "rosca bíceps"15,22-23 - apenas essa informação pode gerar dúvidas em relação a sua execução. Indicaçôes como o tipo de empunhadura realizada e equipamento a ser utilizado facilitariam seu entendimento e sua prescrição.

Como fatores limitantes do trabalho apresentado, podem ser citados o número de indivíduos entrevistados, que 
pode ser considerado baixo levando-se em consideração o número de profissionais que atuam com TF. Outro fator limitante tange a questáo do estudo ter sido realizado somente por profissionais e estudantes que atuam no estado do Rio de Janeiro. Contudo, estudos futuros podem ser desenvolvidos em uma área geográfica mais extensa e com um número maior de voluntários pesquisados, bem como outros tipos de exercícios podem ser analisados, como os realizados com implementos e acessórios.

\section{Conclusão}

Diante do apresentado, foi possível observar um consenso nas nomenclaturas na maioria dos exercícios analisados. Adicionalmente, foi possível verificar que não houve relação entre o TETF e os nomes adotados pelos profissionais e estudantes de educação física. Tendo em vista o exposto, padronizar nomenclaturas para os exercícios pode ser uma estratégia positiva para os profissionais que trabalham na área de TF, beneficiando a comunicação e compreensão dos exercícios prescritos em programas de treinamento frequentemente utilizados em academias de ginástica.

\section{Referências}

1. Hall SJ. Biomecânica básica. $4^{\mathrm{a}}$ ed. Rio de Janeiro: Guanabara Koogan; 2005.

2. Jackson MC, Brown LE, Coburn JW, Judelson DA, CullenCarroll N. Towards standardization of the nomenclature of resistance training exercises. J Strength Cond Res. 2013;27(5):1441-9.

3. Souza JAAA, Nunes MB, Paz GA, Miranda HL. Nomenclatura dos exercícios estabilizadores de fortalecimento do método pilates: existe padronização? Rev Bras Ciênc Mov. 2016;24(1):145-52.

4. Souza LML, Paz GA, Viviane SS, Miranda H. Comparison study of resistance exercise nomenclature adopted among professionals and undergraduate physical education students. Rev Bras Cineantropom Desempenho Hum. 2016;18(2):233-42.

5. Nuzzo JL. The words and patterns that comprise resistance training exercise names. J Strength Cond Res. 2015; abr. 7. [Epub ahead of print].

6. Fleck SJ, Kraemer WJ. Designing resistance training programs. 3a ed. Champaign: Human Kinetics; 2004.

7. Faigenbaum AD, Myer GD. Resistance training among young athletes: safety, efficacy and injury prevention effects. Brit J Sports Med. 2010;44(1):56-63.

8. Faulkner JA. Terminology for contractions of muscles during shortening, while isometric, and during lengthening. J Appl Physiol. 2003;95(2):455-9.
9. National Strength and Conditioning Association. Exercise technique manual for resistance training. Champaign: Human Kinetics; 2008.

10. Betti R, Betti M. Novas perspectivas na formação profissional em educação física. Motriz Rev Educ Fís. 1996;2(1):10-5.

11. Vale RGS, Novaes JS, Dantas EHM. Efeitos do treinamento de força e de flexibilidade sobre a autonomia de mulheres senescentes. Rev Bras Ciênc Mov. 2005;13(2):33-40.

12. Mediano MFF, Paravidino V, Simão R, Pontes FL, Polito MD. Comportamento subagudo da pressão arterial após o treinamento de força em hipertensos controlados. Rev Bras Med Esporte. 2005;11(6):337-40.

13. Gonçalves R, Gurjão ALD, Gobbi S. Efeitos de oito semanas do treinamento de força na flexibilidade de idosos. Rev Bras Cineantropom Hum. 2007;9(2):145-53.

14. Terra DF, Mota MR, Rabelo HT, Bezerra LMA, Lima RM, Ribeiro AG, et al. Redução da pressão arterial e do duplo produto de repouso após treinamento resistido em idosas hipertensas. Arq Bras Cardiol. 2008;91(5):299-305.

15. Okano AH, CyrinoES, Nakamura FY, Guariglia DA, Nascimento MA, Avelar A, et al. Comportamento da força muscular e da área muscular do braço durante 24 semanas de treinamento com pesos. Rev Bras Cineantropom Hum. 2008;10(4):379-85.

16. Lagoeiro CG, Silva NS, Robert-Pires CM, Magosso RF. Índice de força máxima relativa de homens treinados nos exercícios puxador costas, puxador frente, pull down e remada unilateral. Rev Bras Prescr Fisiol Exerc. 2014;8(44):156-62.

17. Cunha MF, Lazzareschi L, Gantus MC, Suman MR, Silva A, Parizi CC, et al. A influência da fisioterapia na prevenção de quedas em idosos na comunidade: estudo comparativo. Motriz. 2009;15(3):527-36.

18. 18. Lima PFM, Araújo RC, Farah BQ, Cavalcante BR, Santos MAM, Ritti-Dias RM. Reprodutibilidade do teste de uma repetição máxima em exercícios de força com pesos livres. Rev Bras Ativ Fís Saúde. 2013;18(3):378-80.

19. Calais-Germain B. Anatomia para o movimento: vol. 1: introdução à análise das técnicas corporais. $1^{\mathrm{a}} \mathrm{ed}$. São Paulo: Manole; 2002.

20. Kapandji AI. Fisiologia articular: esquemas comentados de mecânica humana: vol. 1. $5^{\mathrm{a}}$ ed. São Paulo: Panamericana; 2000.

21. Barbanti VJ, Tricoli V, Ugrinowitsch C. Relevância do conhecimento científico na prática do treinamento físico. Rev Paul Educ Fís. 2004;18:101-9.

22. Polito MD, Simão R, Senna GW, Farinatti PTV. Efeito hipotensivo do exercício de força realizado em intensidades diferentes e mesmo volume de trabalho. Rev Bras Med Esporte. 2003;9(2):69-73.

23. Salvador EP, Cyrino ES, Gurjão ALD, Ritti-Dias RM, Nakamura FY, Oliveira AR. Comparaçáo entre o desempenho motor de homens e mulheres em séries múltiplas de exercícios com pesos. Rev Bras Med Esporte. 2005;11(5):257-61.

\section{Como citar este artigo:}

Souza LML, Paz GA, Straatmann VS, Miranda H. Associaçóes entre o tempo de experiência profissional e a nomenclatura dos exercícios utilizados no treinamento de força. Rev. Aten. Saúde. 2016;14(50):49-55. 